\title{
Infrared Sensitivity of Unstable Vacua
}

\author{
Dmitry Krotov, Alexander M. Polyakov \\ Joseph Henry Laboratories, Princeton University, Princeton, NJ 08544, USA
}

\begin{abstract}
We discover that some unstable vacua have long memory. By that we mean that even in the theories containing only massive particles, there are correllators and expectation values which grow with time. We examine the cases of instabilities caused by the constant electric fields, expanding and contracting universes and, most importantly, the global de Sitter space. In the last case the interaction leads to a remarkable UV/IR mixing and to a large back reaction. This gives reasons to believe that the cosmological constant problem could be resolved by the infrared physics.
\end{abstract}

\section{Introduction}

The problem of cosmological constant presents a serious challenge to modern physics. Recently the following physical mechanism was proposed to overcome this problem 2. Let us imagine that the bare cosmological constant is present in the lagrangian. As is well known, it is the cause of gravitational repulsion, resulting in the accelerated expansion of the universe (the contraction is also possible, but we will discuss it later). According to the proposal, this acceleration leads to the explosive particle production. The gravitational attraction between the particles slows down the acceleration and thus reduces (asymptotically to zero) the effective cosmological constant.

There are many puzzles associated with this proposal. Particles in the curved space are ill defined, does it make sense to ascribe to them a real physical effect? Even if it does, the Universe is exponentially expanding, so it may seem that the particles get diluted; isn't their back reaction negligible? How can massive particles considered in [2] lead to large infrared effects?

In this paper we will try to provide some clarifications, as well as present some new results. It is helpful to consider various cases of unstable vacua and to make their comparative studies. The basic origin of the difficulties lies in the non-equilibrium quantum field theory and are common to all the cases. Let us demonstrate this with the following example.

Consider a nucleus with a charge $Z$ and hit it with a $\gamma$-quantum of energy $\omega$ which produces a pair $e^{+} e^{-}$. The electron forms a bound state while the positron escapes to infinity. The threshold singularity in $\omega$ is located at

$$
\omega=2 m-\left|E_{B}\right|
$$

where $\left|E_{B}(Z)\right|$ is the binding energy. We see that when the nucleus becomes supercharged, $\left|E_{B}(Z)\right|=2 m$, we get a long-ranged correlations in time since the threshold is now at $\omega=0$. 
Below we will find that such "long-memory" is a crucial factor in the non-equilibrium dynamics. In field theory it leads to the "adiabatic catastrophe" [1] and to the obstruction to Wick's rotation. In the next section we shall briefly summarize the situation.

Another puzzle mentioned above is the dilution of particles in the expanding universe, the size of which grows as $a(t) \sim e^{t}$. However, in any physical quantity, this effect always cancels with the exponentially growing number of the comoving modes. The covariant cut-off for the comoving momentum $k$ is given by $k \leq k_{\max } \sim M_{\text {planck }} a(t)$, and this causes the growth. The naive reason for the above compensation is that the change $a \rightarrow \lambda a$ is a coordinate transformation and $a$ dependence can't be physical. As will be discussed below, there are caveats to this argument, but on a qualitative level they are unimportant.

What about the infrared effects generated by the massive particles? They are not related to the interaction of these particles, which is short-ranged as usual. Their origin lies in the fact that the original vacuum is unstable with the non-zero decay rate. Therefore we get perturbative corrections containing secular terms, which represent the fact that, as the time goes, it is less and less likely for the vacuum to remain intact.

\section{Lorentzian vs Euclidean calculations}

A crude example of these phenomena is provided by a hot plasma in a box. One can calculate various correlations using the Euclidean approach by introducing Matsubara's periodic time. The resulting physical quantities will describe a well-defined physics of plasma kept at fixed temperature by the external sources. However, if such sources are absent, the plasma will cool off. In this case the "Euclidean" calculation is inadequate and we have to use the Schwinger-Keldysh approach.

Similar situation exists in the $d S$ space. This space is defined by the equation

$$
\vec{n}^{2}-n_{0}^{2}=1
$$

It is tempting to define the following rules of the game. Make a "Wick rotation", $n_{0} \Rightarrow i n_{0}$, which transforms the $d S$ space into a sphere. On a sphere massive particles will never generate any IR divergences, at least perturbatively. Suppose now that we calculate the correlation functions $<\varphi\left(n_{1}\right) \ldots \varphi\left(n_{N}\right)>$ on a sphere and then continue them back to the $d S$ space. Such a strategy was briefly considered and rejected in [2, but taken as a fundamental definition of the theory in [12. Moreover, it was shown in these two papers that the analytic continuation from a sphere doesn't lead to any pathologies.

Our claim is that, just as in the above case of plasma, this "Euclidean" approach describes the de Sitter space artificially kept at fixed Gibbons-Hawking temperature. This can be achieved by surrounding the $d S$ patch by reflecting walls sending all emitted radiation back. However without these magic devices Euclidean approach is inadequate.

A very similar situation occurs in the case of the Schwarzschild black holes - their Euclidean geometry is the well-known cigar and the field theory on the cigar describes eternal black hole in which the Hawking radiation is being returned to keep the equilibrium. Let us notice also that in the case of the black hole the Euclidean approach uses $r \geq 2 M$ region where (after Wick's rotation) the killing vectors are positive.

As is well known, its Euclidean metric

$$
d s^{2}=\left(1-\frac{2 M}{r}\right) d t^{2}+\frac{d r^{2}}{\left(1-\frac{2 M}{r}\right)}+r^{2} d \Omega
$$

is complete and non-singular if $t \sim t+8 \pi M$. It has a geometry of a cigar. According to Gibbons and Hawking, periodicity in imaginary time is the indication of Hawking's temperature. An interacting 
field theory on this manifold is also well defined. We can get a set of Green's functions by an analytic continuation to real time.

However, as well known, these continuation will describe the black hole on "life support" its temperature must be kept constant. The real black hole evaporates and can't be described by Wick's rotation. The same is true for the dS space. A typical puzzle here is that while the black hole evaporates into the outer space, the dS space has nowhere to go. In fact, this puzzle is psychological. The dS space simply creates an avalanche of particles within itself. One can also visualize this by immersing a large patch of the dS space in the Minkowski space-time. In this case the created particles will populate the ambient space. For the proper description of the $d S$ space one must use the Schwinger-Keldysh approach with real time.

In the case of the Bunch-Davies vacuum there are some further puzzles. The wave functional in this case is of the Hartle-Hawking type:

$$
\Psi_{0}[\varphi(\vec{n})]=\int_{\left.\varphi\right|_{\partial M}=\text { fixed }} D \varphi e^{-S(\varphi)}
$$

where we integrate over fields on a hemisphere. The correlators are given by

$$
G=\left(\psi_{0}, \varphi\left(n_{1}\right), \ldots, \varphi\left(n_{N}\right) \psi_{0}\right) .
$$

The $d S$ vector $n$ can be parameterized as $n=(\sinh t,(\cosh t) \vec{n})$, the southern hemisphere is described by $-i \pi / 2 \leqslant t \leqslant 0$, while the northern one corresponds to $i \pi / 2 \geqslant t \geqslant 0$. If we consider a perturbation theory analytically continued to $d S$ we have formally

$$
G_{(\text {Euclidean })}=\left(\psi_{0}, T_{C} \varphi\left(n_{1}\right) \ldots \varphi\left(n_{N}\right) e^{-\frac{i \lambda}{4 !} \int \varphi^{4}} \psi_{0}\right)
$$

where the contour $C$ for the correllators on a sphere is shown at Fig.1 left, while the contour after analytic continuation to $d S$ space is shown on the right. These contours must be used

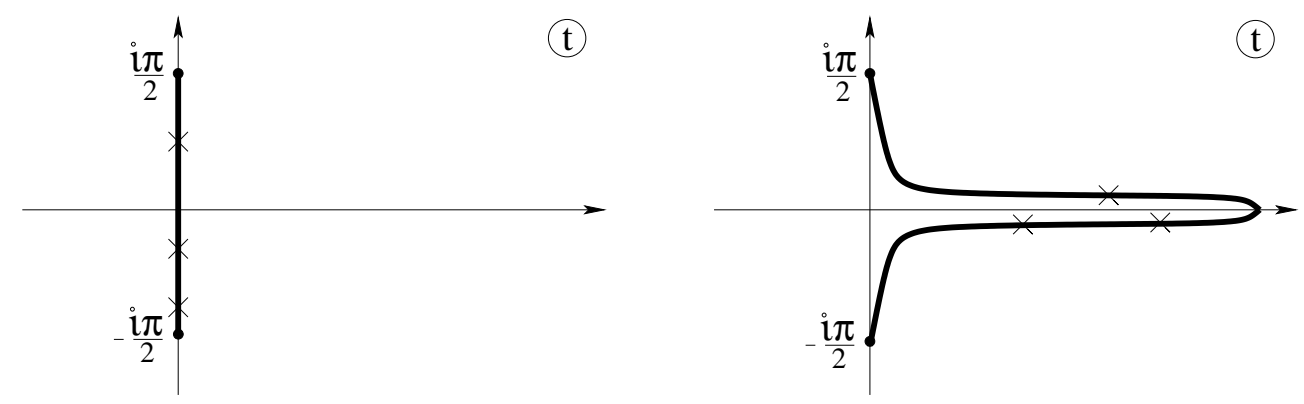

Figure 1: The contours of integration on a sphere and after analytic continuation to $d S$ space.

in the Schwinger-Keldysh diagrams. One might think that this should give the same result as the standard Schwinger-Keldysh contour for the Lorentzian approach, Fig.2.

This is not the case. The reason for the discrepancy lies in the adiabatic catastrophe. We have to introduce the adiabatic switching of the interaction and let the system develop freely:

$$
G_{(\text {Lorentzian })}\left(n_{1}, \ldots\right)=\left\langle 0\left|T \varphi\left(n_{1}\right) \ldots \varphi\left(n_{N}\right) e^{-i \int \lambda\left(\varepsilon n_{0}\right) \varphi^{4} d n}\right| 0\right\rangle
$$

where $\varepsilon \rightarrow 0$.

As we will show below the IR cut-off $\varepsilon$ does not disappear and that implies the instability of the $d S$ space. The reason for the breakdown of adiabaticity is that there are states with negative energies and so the arbitrary slow external field can trigger pair production. 


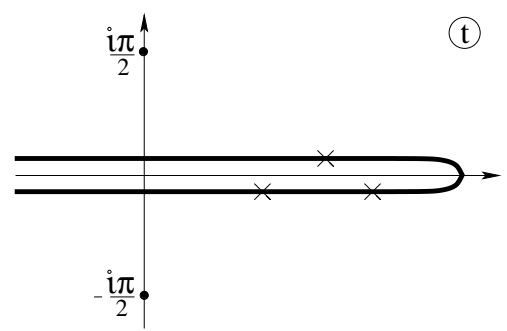

Figure 2: Standard contour of integration in Lorentzian approach.

\section{Electric fields and new anomaly}

Pair production by electric fields has been discussed in the hundreds of valuable papers, see references in [3]. We return to this problem for two reasons. First, we need to present it in the form, which can be easily generalized to the gravitational case. Second, we will find an unusual anomalous vacuum polarization which may have unexpected applications.

Let us consider massive scalar field in electric field, described by a time-dependent vector potential $A_{1}(t)$. We assume that electric field is switched on and off adiabatically. This means that it has the form $E=E\left(\frac{t}{T}\right)$ so that for $|t|<<T$, it remains constant while for $|t|>>T$, $E \rightarrow 0$. A good concrete example of such behavior (already considered in 4]) is to take

$$
A_{1}(t)=E T \tanh \left(\frac{t}{T}\right)
$$

$E(t)=\frac{E}{\cosh \left(\frac{t}{T}\right)^{2}}$, but explicit shape of the potential is not important. The Klein-Gordon equation has the form

$$
\left(\partial_{t}^{2}+(k-A(t))^{2}+k_{\perp}^{2}+m^{2}\right) \varphi=0
$$

We are interested in the 'in' solution which is defined as the 'Jost function', i.e. it has the asymptotic behavior

$$
\varphi_{i n}(t, k) \rightarrow_{t \rightarrow-\infty} \frac{1}{\sqrt{2 \omega_{k}^{-}}} e^{-i \omega_{k}^{-} t}
$$

where $\omega_{k}^{ \pm}=\sqrt{(k-A( \pm \infty))^{2}+k_{\perp}^{2}+m^{2}}$. The solution is normalized by the condition that Wronskian $W\left(\varphi, \varphi^{*}\right)=1$.

As we go to the late time $t \rightarrow \infty$, we have

$$
\varphi_{i n}(t, k) \rightarrow_{t \rightarrow \infty} \frac{1}{\sqrt{2 \omega_{k}^{+}}}\left[\alpha(k) e^{-i \omega_{k}^{+} t}+\beta(k) e^{i \omega_{k}^{+} t}\right]
$$

where $\alpha$ and $\beta$ are Bogolyubov coefficients also related to the transmission and reflection amplitudes.

If we start with $\varphi_{\text {in }}$ and blindly apply the WKB approximation, we get

$$
\varphi_{\text {in }}(t, k) \sim \frac{1}{\sqrt{2 \omega_{k}(t)}} e^{-i \int_{0}^{t} \omega_{k}\left(t^{\prime}\right) d t^{\prime}}
$$

for late times, with $\omega_{k}(t)=\sqrt{(k-A(t))^{2}+k_{\perp}^{2}+m^{2}}$. Of course in this way we loose the over barrier reflection and thus the above formula can't be valid everywhere. Indeed the WKB requires 
that the de Broglie wave length $\lambda=\frac{1}{\omega_{k}}$ satisfies

$$
\gamma=\frac{d \lambda}{d t}=\frac{(k-A) \dot{A}(t)}{\left[(k-A)^{2}+k_{\perp}^{2}+m^{2}\right]^{\frac{3}{2}}} \ll 1
$$

We see that the approximation is good for the early times when $|k-A(t)| \gg m$. In this case

$$
\gamma \sim \frac{E}{|k-A|^{2}} \sim \frac{m^{2}}{|k-A|^{2}} \frac{E}{m^{2}} \ll 1
$$

if we assume $E \sim m^{2}$.

However, around the point where the mode 'reaches the horizon', defined by $k=A\left(t_{k}\right)$, we get $\gamma \sim 1$ and WKB breaks down. As we go to $t \gg t_{k},|k-A|$ starts growing again and the WKB is valid again. In this region it must contain two exponentials:

$$
\varphi_{i n}(t, k) \sim \frac{1}{\sqrt{2 \omega_{k}(t)}}\left[\alpha(k) e^{-i \int_{0}^{t} \omega_{k}}+\beta(k) e^{i \int_{0}^{t} \omega_{k}}\right] \quad t \gg t_{k}
$$

As usual, $\alpha$ and $\beta$ can be found by matching (2) and (5).

In the domain $|t| \ll T$ the electric field is constant and $A(t) \sim E t$. The equation (1) now depends on the variable $t-\frac{k}{E}$, hence $\varphi_{i n} \sim f_{i n}\left(t-\frac{k}{E}\right)$. The function $f_{i n}$, as well known, is the parabolic cylinder function

$$
\varphi_{i n} \sim D_{-\frac{1}{2}-i \lambda}\left[-\sqrt{2 E} e^{i \frac{\pi}{4}}\left(t-\frac{k}{E}\right)\right] \quad t \rightarrow-\infty
$$

but we will not need their explicit form. What is important is that due to the symmetry $k \rightarrow k+\kappa$, $t \rightarrow t-\frac{\kappa}{E}$ the resulting $\alpha$ and $\beta$ do not depend on $k$ in a certain range, which we determine in a moment (but do depend on $k_{\perp}$ and $m$ ).

To find this range we notice that the 'horizon crossing' $(k=A(t))$ occurs at $t_{k}=\frac{k}{E}$. We can use the constant field approximation only if $t_{k} \ll T$. Hence we conclude that $\alpha$ and $\beta$ do not depend on $k$ only if $A(-\infty)<k<A(\infty)$. Outside this interval reflection coefficient $\beta$ quickly decreases to zero.

The field $\varphi$ can be expanded in terms of creation and annihilation operators as

$$
\varphi=\sum_{k}\left(a_{k} f_{k}^{i n *} e^{i k x}+b_{k}^{\dagger} f_{k}^{i n} e^{-i k x}\right)
$$

and the Green function is equal to

$$
\begin{aligned}
G\left(x_{1}, t_{1} \mid x_{2}, t_{2}\right)={ }_{i n}\left\langle 0\left|T \varphi\left(x_{1}, t_{1}\right) \varphi\left(x_{2}, t_{2}\right)^{*}\right| 0\right\rangle_{i n} & =\int f_{k}^{i n}\left(t_{<}\right) f_{k}^{i n *}\left(t_{>}\right) e^{i k\left(x_{1}-x_{2}\right)} d k= \\
& =e^{i E \frac{t_{1}+t_{2}}{2}\left(x_{1}-x_{2}\right)} g\left(t_{1}-t_{2}, x_{1}-x_{2}\right)
\end{aligned}
$$

The first factor here is a gauge dependent phase which must cancel in physical quantities. The remaining part $g\left(t_{1}-t_{2}, x_{1}-x_{2}\right)$ is invariant under translations and defines correlation functions of gauge invariant quantities. In particular we can calculate the induced current which can be used to estimate the back reaction. The general formula for the current is

$$
\langle J(t)\rangle=\int(k-A(t))\left|\varphi_{i n}(k, t)\right|^{2} d k
$$


As we will see, the current is dominated by the two semi-classical domains described above. Before the 'horizon crossing' we have

$$
\langle J(t)\rangle^{(1)}=\int_{A(t)<k} d k d k_{\perp} \frac{(k-A(t))}{2 \omega_{k}(t)}=\int_{0}^{\infty} \frac{d p p d k_{\perp}}{2 \sqrt{p^{2}+k_{\perp}^{2}+m^{2}}}
$$

where $p=k-A(t)$ is 'physical momentum'. After horizon crossing, we have to use (3). Keeping only non-oscillating terms, which are dominant, we obtain

$$
\langle J\rangle^{(2)}=\int_{k<A(t)} d k d k_{\perp} \frac{k-A(t)}{\sqrt{(k-A)^{2}+k_{\perp}^{2}+m^{2}}}\left(|\alpha(k)|^{2}+|\beta(k)|^{2}\right)
$$

Using the general relation $|\alpha(k)|^{2}-|\beta(k)|^{2}=1$ we get:

$$
\langle J\rangle^{(2)}=\int_{-\infty}^{0} \frac{d p d k_{\perp} p}{2 \sqrt{p^{2}+k_{\perp}^{2}+m^{2}}}+2 \int_{-\infty}^{0} \frac{d p d k_{\perp}\left|\beta\left(k, k_{\perp}\right)\right|^{2} p}{2 \sqrt{p^{2}+k_{\perp}^{2}+m^{2}}}
$$

The first term in this formula combines with (6) and gives zero due to $p \rightarrow-p$ symmetry. The second term is really interesting. The key feature of it is that the reflection coefficient $\beta$ depends on the 'comoving' momentum $k$ and not the physical one $p$. As we saw, this coefficient keeps being constant for $A(-\infty) \ll k \ll A(\infty)$ and quickly vanishes outside this interval. In terms of $p$, this means the time-dependent cut-off $A(-\infty) \ll p+A(t) \ll A(\infty)$. We also have a cut-off on $k_{\perp}$, $k_{\perp} \ll E$. Hence, the total current is given by

$$
\langle J\rangle=\int_{A(-\infty)-A(t)}^{0} d p \frac{p}{|p|} \int d k_{\perp}\left|\beta\left(k_{\perp}, k\right)\right|^{2}=-(A(t)-A(-\infty))|\beta|^{2} E^{\frac{d-1}{2}} \cdot \text { const }
$$

In the last expression $|\beta|^{2}=e^{-\frac{\pi m^{2}}{E}}$. This result is physically transparent. It means that, as the time goes by, more and more $k$ modes cross the horizon $k=A(t)$ and begin to contribute to the induced current. This fact is important. It shows that the induced current is proportional to the vector potential and not the field strength. Together with gauge invariance this implies highly non-local behavior. Indeed, $A(t)-A(-\infty)=\int_{-\infty}^{t} d t^{\prime} E\left(t^{\prime}\right)$. Similar non-localities are well known - the London equation in superconductors, the photon mass in the Schwinger Model, the Chern-Simons terms in the quantum Hall effect. In all these cases the gauge invariant expressions can't be expressed locally in terms of the the field strengths.

The stunning feature of the above result is that the non-locality appears in the massive theory. This is specific for unstable vacua and can't be seen in the in/out formalism. It also implies the strong back reaction, since the current is growing with time. Another interpretation of this result is symmetry breaking. Indeed, in the constant field we obviously have the time translation invariance. This invariance is broken in the expression for the current due to the influence of the past when the field was turning on. We will return to this phenomenon later, while discussing the gravitational case.

In the mean field approximation we plug the current back into Maxwell's equation similarly to the procedure of [5] 1 . Considering for simplicity $1+1$ dimensions we get

$$
\ddot{A}=J=-2|\beta(\dot{A})|^{2} A(t)
$$

\footnotetext{
${ }^{1}$ In the recent paper $[7$ the in/in current has been calculated. The result is different from ours and we disagree with the method used in this paper. Another expression for the in/in current, which is consistent with our result, can be found in [8]. Let us also notice the paper [9] on R-N black holes which used the method very close to ours.
} 
At large time the solution decays as $E=\dot{A} \sim \frac{1}{\log t}$ indicating the total screening of the electric field. This may be related to the old result by Gribov [6]. Another analogy is the Landauer conductance in the mesoscopic system, which is also expressed in terms of the transmission and reflection coefficients.

Another interesting point is that for massless fermions the anomaly equation reads as

$$
\partial_{0} J_{1}=\frac{1}{\pi} E
$$

since axial and vector currents are related by epsilon symbol in two dimensions. This gives linear growth of current with time and is consistent with our result since in massless case $\beta \sim 1$. The back-reaction can be estimated from the Maxwell equation $\dot{E}+J_{1}=0$ and is clearly significant. In the case of fermions (the Schwinger Model) we know that in the vacuum no electric field remains and electric charges are completely screened.

We can also use the in/out Green function

$$
G^{\text {in } / \text { out }}=\frac{1}{\alpha} \varphi_{k}^{\text {in }}\left(t_{<}\right) \varphi_{k}^{\text {out } *}\left(t_{>}\right)
$$

The sign of vacuum instability here is $\operatorname{Im} G(t \mid t) \neq 0$. Let us notice that the matrix element $\left\langle\right.$ out $\left|J_{1}\right|$ in $\rangle=0$ simply because the in/out Green function is Lorentz invariant (module a phase factor). The Euclidean version of this phenomenon (with the replacement of the electric field by the magnetic one) is the absence of bulk currents in the quantum Hall effect.

It is also instructive to change the gauge. If we take $A_{0}=E z$ we get Klein-Gordon equation

$$
\left(\partial_{z}^{2}+(\omega-E z)^{2}-m^{2}\right) \varphi=0
$$

As in the time-dependent gauge, we have a Schrodinger equation for inverted oscillator, but this time the effect of pair creation comes from the underbarrier penetration rather than from the overbarrier reflection. The two are related by the analytic continuation. In this gauge the energy $\omega=E z+\sqrt{p^{2}+m^{2}}$ is conserved but non-positive which allows particle production. This gauge has de Sitter counterpart. The tunneling above is analogous to the tunneling in the Painleve coordinates [10].

\section{Expanding Universe (free fields)}

When we look at the de Sitter space, we find that there are striking similarities with the electric case. Let us consider what happens when the curvature of $d S$ space is adiabatically switched on. In this setting we have two quite different problems - expanding and contracting universes. The arrow of time is set up by defining the infinite past as a Minkowski space in which our field is in the ground state and solutions to the wave equation are chosen to be the Jost functions. Let us begin with the expanding Universe. Analogously to the electric case we will assume that the FRW metric

$$
d s^{2}=a(t)^{2} d \vec{x}^{2}-d t^{2}
$$

is such that $\frac{\dot{a}}{a}=H\left(\frac{t}{T}\right)$, time $T$ is supposed to be large, and $H(0)=1$, while $H( \pm \infty)=0$. A representative example of such a metric is

$$
a(t)=e^{T \tanh \frac{t}{T}}
$$

$H(t)=\frac{1}{\cosh \left(\frac{t}{T}\right)^{2}}$. It is convenient to rescale the standard scalar field $\varphi$ by defining $\varphi=a^{-\frac{d}{2}} \phi$. The Klein-Gordon equation takes the form

$$
\ddot{\phi}_{i n}+\left(m^{2}-r(t)+\frac{k^{2}}{a(t)^{2}}\right) \phi_{i n}=0
$$


with $r(t)=\frac{d(d-2)}{4}\left(\frac{\dot{a}}{a}\right)^{2}+\frac{d}{2} \frac{\ddot{a}}{a}$. As before, the 'in' solution is defined by

$$
\phi_{i n}=\frac{1}{\sqrt{2 \omega_{k}^{-}}} e^{-i \omega_{k}^{-} t}
$$

as $t \rightarrow-\infty$ with $\omega_{k}^{-}=\left(m^{2}+\frac{k^{2}}{a(-\infty)^{2}}\right)$. Its quasiclassical expression is given by the formula (4) where $\omega_{k}(t)=\sqrt{m^{2}-r(t)+\frac{k^{2}}{a(t)^{2}}}$. This WKB expression is applicable if

$$
\gamma=\dot{\lambda}=\frac{d}{d t}\left(\frac{1}{\omega_{k}}\right) \sim \frac{1}{\left(m^{2}-r+\frac{k^{2}}{a^{2}}\right)^{\frac{3}{2}}} \frac{k^{2}}{a^{2}} \frac{\dot{a}}{a} \ll 1
$$

If we assume that $H=\frac{\dot{a}}{a} \sim m$ and $\dot{H}$ is small, we see that WKB breaks down when the given mode crosses the horizon, $k \sim m a(t)$. Before that we had $k \gg m a(t)$ and $\dot{\lambda} \ll 1$. Long after that we reach the semi-classical regime again, but with two exponentials as in (3). Let us consider the time evolution of the quantity $\left\langle i n\left|\varphi(t)^{2}\right| i n\right\rangle$. We have:

$$
\left\langle i n\left|\varphi(t)^{2}\right| i n\right\rangle=\int d^{d} k\left|\varphi_{i n}(t, k)\right|^{2}
$$

splitting the integral as before into the regions $|k| \gg m a(t)$ and $|k| \ll m a(t)$ we get

$$
\begin{aligned}
\left\langle i n\left|\varphi(t)^{2}\right| i n\right\rangle & =a(t)^{-d} \int_{|k| \gg m a(t)} \frac{d^{d} k}{2 \omega_{k}(t)}+a(t)^{-d} \int_{|k| \ll m a(t)} \frac{d^{d} k}{2 \omega_{k}(t)}\left[|\alpha(k)|^{2}+|\beta(k)|^{2}\right]= \\
& =a^{-d}\left(\int \frac{d^{d} k}{2 \omega_{k}(t)}+2 \int_{|k| \ll m a(t)} \frac{d^{d} k}{2 \omega_{k}(t)}|\beta(k)|^{2}\right)
\end{aligned}
$$

The reflection amplitude $\beta(k)$ is $k$-independent in a certain interval, just as it was in the electric case. The reason is that de Sitter wave equation is invariant under $k \rightarrow \lambda k$ and $t \rightarrow t+\log \lambda$ (which is one of the $d S$ isometries). However this amplitude quickly vanishes when $k$ is such that the horizon crossing happens outside the de Sitter stage. Namely, if $t_{k}$ is determined from the equation $k=m a\left(t_{k}\right)$, the constant reflection occurs for $\left|t_{k}\right| \ll T$. If we introduce the cutoffs defined by $\frac{k_{\min }}{a(-\infty)}=k_{\min } e^{T}=m$ and $\frac{k_{\max }}{a(+\infty)}=k_{\max } e^{-T}=m$, we have reflection only if $k_{\min } \ll k \ll k_{\max }$. We see that the contribution of the second term in (9), which represents the created particles, is small in the expanding case. Due to the infrared convergence of the integral we obtain

$$
\left\langle\varphi(t)^{2}\right\rangle^{(2)} \sim|\beta|^{2} m^{d-1}
$$

This formula has a clear physical interpretation. By the moment $t$ we excite the modes with $|k|<m a(t)$ and the average excitation number is $\bar{n} \sim|\beta|^{2}$. The created particles are nonrelativistic due to the upper boundary on $k$. Let us stress that there is no dilution of the created particles in the sense that their physical (not comoving) density remains constant in time, however their main contribution is just a renormalization of the cosmological constant which is unobservable.

The key difference from the electric case is the absence of the dynamical symmetry breaking, which we define as a long-term memory. By this we mean the following. As we already noticed, the current in the electric case depends on the time passed from the first appearance of the field. This effect is a dynamical counterpart of the usual spontaneous symmetry breaking. In the latter case, the magnetic field at the boundary induces magnetic moment in the bulk, if we talk about 
Ising model for example. In our case the role of the boundary is played by the infinite past. The expression (10) does not depend on time. Hence, there is no dynamical breaking of de Sitter symmetry in this case. Life becomes more interesting if we switch on interaction or consider contracting universe.

We could calculate things in the regime of the constant curvature and get the right results. In this case

$$
\varphi_{i n} \sim \tau^{\frac{d}{2}} H_{i \mu}^{(1)}(k \tau)
$$

with $\tau=e^{-t}$ and

$$
\left\langle i n\left|\varphi(t)^{2}\right| i n\right\rangle \sim \tau^{d} \int d^{d} k\left|H_{i \mu}^{(1)}(k \tau)\right|^{2}=\int d^{d} p\left|H_{i \mu}^{(1)}(p)\right|^{2}=\text { const }
$$

The UV divergence in this integral is the same as in the flat space and the time independence in this formula is just the result of the de Sitter symmetry. The back reaction is thus small and uninteresting. Really non-trivial things begin to happen when we either include interactions or consider contracting universe. We start with the latter.

\section{Contracting Universe (free fields)}

Let us repeat the above calculations in the case of contracting Universe2 2 . At the first glance it may seem that, since de Sitter space is time-symmetric, expansion and contraction can't lead to different results. However, as was stated above there is an arrow of time in our problem. We defined the past by the condition that our field is in the Minkowsky vacuum state. Generally speaking, in the future we should expect complicated excited state. In this setting contraction is very different from expansion. We can once again take

$$
a(t)=e^{-T \tanh \frac{t}{T}}
$$

The modes with $k>m a(-\infty)=m e^{T}$ will always stay in the WKB regime, since $a(t)$ will be decreasing. On the other hand, the modes with $m a(\infty) \ll k \ll m a(-\infty)$ will cross the horizon at some time, $k \approx m a\left(t_{k}\right)$. If we once again define the 'in' modes, $\varphi_{i n}(k, t)$ by the condition (8), we find that for $k \ll m a(t)$ the horizon crossing (WKB breaking) has not occurred yet (remember that $a(t)$ is decreasing) and hence we have a single exponential (8).

For $m a(t) \ll k \ll m a(-\infty)$ the horizon crossing is already in the past and we have two exponentials with the coefficients $\alpha$ and $\beta,|\alpha(k)|^{2}-|\beta(k)|^{2}=1$. For $k \gg m a(-\infty)$, the horizon crossing has never occurred and $\beta \rightarrow 0$. As in the previous section we get

$$
\begin{aligned}
\left\langle i n\left|\varphi(t)^{2}\right| i n\right\rangle & =a(t)^{-d} \int_{|k| \ll m a(t),} \frac{d^{d} k \mid \gg m a(-\infty)}{2 \omega_{k}(t)}+a(t)^{-d} \int_{m a(t) \ll|k| \ll m a(-\infty)} \frac{d^{d} k}{2 \omega_{k}(t)}\left[|\alpha(k)|^{2}+|\beta(k)|^{2}\right]= \\
& =a^{-d}\left(\int \frac{d^{d} k}{2 \omega_{k}(t)}+2 \int_{m a(t) \ll|k| \ll m a(-\infty)} \frac{d^{d} k}{2 \omega_{k}(t)}|\beta(k)|^{2}\right)
\end{aligned}
$$

Collecting different terms we get

$$
\begin{aligned}
\left\langle\varphi(t)^{2}\right\rangle & =a^{-d} \int_{|k| \ll \Lambda a(t)} \frac{d^{d} k}{2 \omega_{k}}+2|\beta|^{2} a^{-d} \int_{m a(t)<|k|<m a(-\infty)} \frac{d^{d} k}{2 \omega_{k}(t)} \approx \\
& \approx \text { const } \cdot \Lambda^{d-1}+|\beta|^{2}\left(\frac{a(-\infty)}{a(t)}\right)^{d-1} m^{d-1}
\end{aligned}
$$

\footnotetext{
${ }^{2}$ We considerd the case of contracting universe following the advice of V. Mukhanov.
} 
The first term in this formula is just the same UV divergent term as in the Minkowsky space. The heart of the matter is the second term which displays the symmetry breaking through the long-term memory (dependence on $a(-\infty)$ ). However, the memory can't be too long, since we have a standard UV cut-off at the Planck mass. Because of it, the above formulae are valid if $p=k / a(t)<M_{p l}$ and therefore $a(-\infty) / a(t)<M_{p l} / m$.

Let us sum up the above discussion. In the expanding universe the contribution from the created particles comes from the region $m a(-\infty) \ll k \ll m a(t)$. No long term memory is present and time-dependent back reaction is small, of the order of $\left(\frac{a(-\infty)}{a(t)}\right)^{d-1}$. Created particles are non-relativistic due to the red shift.

In the case of contracting universe particles come from $m a(t)<|k|<\min \left(\operatorname{ma}(-\infty), M_{p l} a(t)\right)$. They are ultra-relativistic and their contribution is of the order $\left(\frac{a(-\infty)}{a(t)}\right)^{d-1} \rightarrow \infty$. All these conclusions are correct only for non-interacting particles.

It is also possible to calculate the energy-momentum tensor. We have

$$
T_{00}=\int d^{d} k\left(\left(\partial_{0} \varphi\right)^{2}+\frac{1}{a(t)^{2}}\left(\partial_{i} \varphi\right)^{2}+m^{2} \varphi^{2}\right)
$$

In the contracting case the order of magnitude of this quantity is defined by the integral:

$$
T_{00} \sim a^{-d} \int \frac{d^{d} k}{2 \omega_{k}} \frac{k^{2}}{a^{2}}|\beta|^{2} \sim a^{-d-1} \int_{m a<k<m a(-\infty)} d^{d} k|k||\beta|^{2} \sim m^{d+1}\left(\frac{a(-\infty)}{a(t)}\right)^{d+1}|\beta|^{2}
$$

This corresponds to the ultra-relativistic particles with the equation of state $p=\frac{1}{d} \varepsilon$. In the expanding case the contribution to $T_{00}$ comes from a small number of created non-relativistic particles. In both cases there are no reasons to believe that created particles are in thermal equilibrium. Let us also stress that the above formula represents a non-local contribution to $T_{00}$ similar to (7). In contrast with this formula, the local contributions should depend on the quantities taken at the time $t$ only.

\section{Secular interactions and the leading logarithms, Poincare patch}

In this section we discuss a very peculiar property of the de Sitter space. Namely, it turns out that the interactions of the massive particles generate infrared corrections. We start with the second order of perturbation theory in the case of $\lambda \varphi^{3}$ interactions (which we choose to simplify notations; the phenomenon we are after is general and has nothing to do with the naive lack of the ground state of the above interaction). We first calculate the correction to the Green's function $G(\vec{q}, \tau)=\langle i n|\varphi(\vec{q}, \tau) \varphi(-\vec{q}, \tau)| i n\rangle$ where $\vec{q}$ is a comoving momentum in the Poincare patch and $\tau$ is a conformal time. Our goal is to show that if the physical momentum $p=q \tau \ll \mu$, there are corrections of the order $\left(\lambda^{2} \log \frac{\mu}{p}\right)^{n}$ where $\mu$ is the particle mass; notice also that these logarithms are the powers of the physical time $t=-\log \tau$.

We are interested in the loop corrections to the one-point function $\left\langle\varphi(t)^{2}\right\rangle$. The magnitude of this quantity determines the strength of the backreaction. To find it we have to use the SchwingerKeldysh perturbation theory. These methods are well known and we will add a few explanations to fix notations. Let us suppress first the momentum dependence and expand $\varphi=f^{*} a+f a^{+}$, where $f(t)$ are the "in" modes and $a$ is an annihilation operator. The relevant one-loop diagram 
is shown at Fig.3. It's contribution to $G(\vec{q}, \tau)=\langle i n|\varphi(\vec{q}, \tau) \varphi(-\vec{q}, \tau)| i n\rangle$ is given by

$$
\begin{array}{r}
G(\vec{q}, \tau)=-\lambda^{2} f_{q}^{*}(t)^{2} \int_{-\infty}^{t} d t_{1} d t_{2} f_{q}\left(t_{1}\right) f_{q}\left(t_{2}\right) \int \frac{d^{d} k}{(2 \pi)^{d}} f_{k}\left(t_{<}\right) f_{k}^{*}\left(t_{>}\right) f_{k+q}\left(t_{<}\right) f_{k+q}^{*}\left(t_{>}\right)-c . c .+ \\
+2 \cdot \lambda^{2}\left|f_{q}(t)\right|^{2} \int_{-\infty}^{t} d t_{1} d t_{2} f_{q}\left(t_{1}\right) f_{q}^{*}\left(t_{2}\right) \int \frac{d^{d} k}{(2 \pi)^{d}} f_{k}\left(t_{1}\right) f_{k}^{*}\left(t_{2}\right) f_{k+q}\left(t_{1}\right) f_{k+q}^{*}\left(t_{2}\right)
\end{array}
$$

In the first line we have the contribution of the $(+/+)$ and $(-/-)$ diagrams (the signs refer to the points $t_{1,2}$ of the physical time, or $\tau_{1,2}$ of conformal time at the diagram in Fig.3), while in the second line we have $(+/-)$ and $(-/+)$ diagrams.

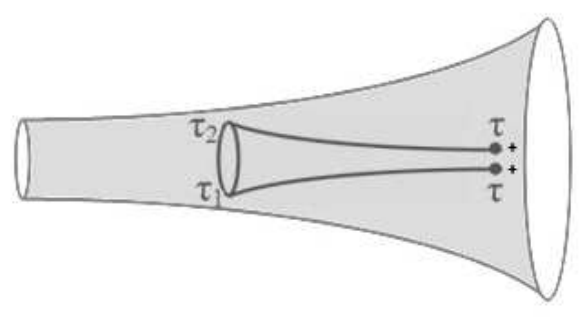

Figure 3: One-loop diagram responsible for infrared logarithms in Poincare patch.

We choose the "in" wave function to be a Hankel function

$$
f_{k}(t)=\tau^{\frac{d}{2}} h(k \tau)=\text { const }^{d / 2} H_{i \mu}^{(1)}(k \tau)
$$

where the normalization is fixed by the condition $h(x) \rightarrow(2 x)^{-\frac{1}{2}} e^{i x}$ as $x \rightarrow \infty$. With this normalization, the asymptotic behavior at $x \rightarrow 0$ is given by

$$
h(x) \rightarrow A(\mu) x^{i \mu}+A(-\mu) x^{-i \mu}
$$

where $A$-s are some concrete functions which we discuss later.

As we will show in a moment, there are infrared logarithmic corrections to $G(\vec{q}, \tau)=\tau^{d} g(q \tau)$ when $q \tau \ll \mu$. In this regime we can use asymptotic expressions (14) to get, $\tilde{\lambda}=\lambda^{2} \log \left(\frac{\mu}{q \tau}\right)$

$$
g(x)=A(\mu) A^{*}(-\mu) \Gamma(\tilde{\lambda}, \mu) x^{2 i \mu}+A(-\mu) A^{*}(\mu) \Gamma^{*}(\tilde{\lambda}, \mu) x^{-2 i \mu}+\left(|A(\mu)|^{2}+|A(-\mu)|^{2}\right) C(\tilde{\lambda}, \mu)
$$

when interaction is off $(\tilde{\lambda}=0)$, coefficients $\Gamma^{(0)}=C^{(0)}=1$. Our goal is to find these quantities at non-zero $\lambda$. We start with the interference term $C$.

In order to obtain the contribution to $g(q \tau)$ we have to integrate the diagrams of Fig.3 over the momentum $k$ and the time variables $t_{1}$ and $t_{2}$. The logarithmic contribution comes from the domain $\tau_{1,2} \sim \mu / k$ and $\mu / \tau \gg k \gg q$. In this domain we get the contribution from the first term in (13) in the form

$$
g(q \tau)_{I}=-2 \lambda^{2} h^{*}(q \tau)^{2} \int d^{d} k \int_{\tau}^{\infty} d \tau_{1} \int_{\tau_{1}}^{\infty} d \tau_{2}\left(\tau_{1} \tau_{2}\right)^{d / 2-1} h\left(q \tau_{1}\right) h\left(q \tau_{2}\right) h^{*}\left(k \tau_{1}\right)^{2} h\left(k \tau_{2}\right)^{2}-c . c .
$$


Taking the limit $q \rightarrow 0$ and interchanging 1 and 2 in the complex conjugate term gives

$$
g(q \tau)_{I}=\int_{q}^{\frac{\mu}{\tau}} \frac{d^{d} k}{k^{d}} C_{I}(\mu)=C_{I} \log \left(\frac{\mu}{q \tau}\right)
$$

Here the coefficient is given by

$$
\begin{aligned}
C_{I} & =-4 \lambda^{2}|A(\mu) A(-\mu)|^{2}\left(|g(\mu)|^{2}+|g(-\mu)|^{2}\right) \\
g(\mu) & =\int_{0}^{\infty} d x x^{d / 2-1+i \mu} h^{2}(x)
\end{aligned}
$$

The second term is treated analogously. It has the form

$$
g(q \tau)_{I I}=2 \lambda^{2} h^{*}(q \tau) h(q \tau) \int d^{d} k \int_{\tau}^{\infty} d \tau_{1} d \tau_{2}\left(\tau_{1} \tau_{2}\right)^{d / 2-1} h^{*}\left(q \tau_{1}\right) h\left(q \tau_{2}\right) h^{*}\left(k \tau_{1}\right)^{2} h\left(k \tau_{2}\right)^{2}
$$

Integration gives another logarithm. Summing these contributions finally gives for the interference term

$$
\left\langle\varphi_{q}^{2}\right\rangle=g(q \tau)_{I}+g(q \tau)_{I I}=2 \cdot(B(\mu)-B(-\mu)) \cdot\left(B(\mu)|g(\mu)|^{2}-B(-\mu)|g(-\mu)|^{2}\right) \cdot \lambda^{2} \log \left(\frac{\mu}{q \tau}\right)
$$

where

$$
B(\mu)=|A(\mu)|^{2}=\frac{1}{4 \mu} e^{\pi \mu} \frac{1}{\sinh (\pi \mu)}
$$

The first multiple here is a Wronskian of the eigenmodes. The second one turns out to be equal to zero. To see this, note that the functions $h(x)$ satisfy $h(x)^{*}=e^{i \frac{\pi}{2}} h\left(e^{i \pi} x\right)$ which implies the following relation for $g(\mu)$ :

$$
|g(\mu)|^{2}=e^{-2 \pi \mu}|g(-\mu)|^{2}
$$

The physical meaning of this equality is detailed balance relation with Gibbons-Hawking temperature for de Sitter space. Combining this with the similar property for $A(\mu)$, we conclude that the one-loop contribution to the coefficient in front of the logarithmic divergence in the interference term is equal to zero $C^{(1)}(\tilde{\lambda}, \mu)=0$.

The next step is to calculate $\Gamma$. Imaginary part of this quantity determines the renormalization of mass $\mu$, which we are not interested in at the moment. The real part is responsible for the imaginary contribution to $\mu$, which is related to the decay rate of the particle. Using similar trick: 3 to those used above we find

$$
\operatorname{Re}\left(\Gamma^{(1)}\right)=\lambda^{2}(B(\mu)-B(-\mu))\left(|g(\mu)|^{2}-|g(-\mu)|^{2}\right) \log \left(\frac{\mu}{q \tau}\right)
$$

This quantity is non-zero and negative.

The above calculation refers to the IR properties of the two-point function. In the case of Poincare patch there is no IR contribution to the one-point quantities, as can be seen from the conformal diagram at Fig.4. The Poincare patch is shown here by the gray area. Interactions contributing to the one-point function must be located inside the past light cone due to causality. Therefore we have to consider only the intersection of the light-cone with the gray area defining Poincare patch. Thus infrared effects in Poincare patch can not have dramatic consequences

${ }^{3}$ It is convenient to rescale $k$ from the integrals over $\tau_{1,2}$ and note that

$$
Y=\int_{0}^{\infty} d x \int_{x}^{\infty} d y(x y)^{\frac{d}{2}-1}\left(\left(\frac{x}{y}\right)^{i \mu}+\left(\frac{x}{y}\right)^{-i \mu}\right) h(y)^{2} h^{*}(x)^{2}=\frac{1}{2}\left(|g(\mu)|^{2}+|g(-\mu)|^{2}\right)+i A
$$

where $A$ is some real number, contributing to renormalization of $\mu$ only. 


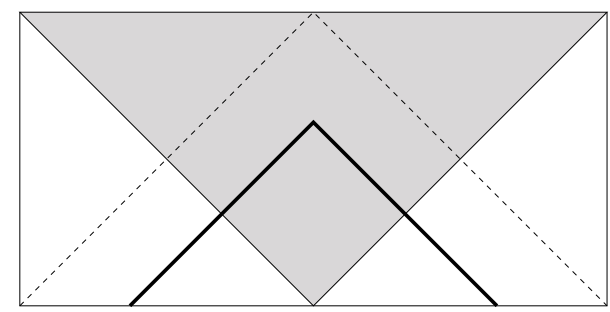

Figure 4: Conformal diagram. Poincare patch is shown by the gray area. Solid black line represents the past light cone of the observer. The intersection of this cone with Poincare patch touches past infinity only at one point.

because the past infinity is represented only by one point. In the complete de Sitter space the situation is quite different and is discussed in the next section.

Although infrared corrections do not appear in the 1-point function $\left\langle\varphi(t)^{2}\right\rangle$, they contribute to the two point function $\langle\varphi(1) \varphi(2)\rangle$. To illustrate this consider the limit when $\tau_{1}=\tau_{2}=\tau$ and $x^{2}=\left(\vec{x}_{1}-\vec{x}_{2}\right)^{2} \gg \tau^{2}$. This corresponds to $z \rightarrow-\infty$ (23). The bare Green's function in this limit is given by

$$
G_{0}(z, \mu)=\frac{1}{\sqrt{-2 z}}\left[N(\mu)(-z)^{i \mu}+N(-\mu)(-z)^{-i \mu}\right]
$$

The exact Green's function is equal td

$$
\begin{aligned}
G(z)=\left[1+\frac{\lambda^{2}}{2}(B(\mu)-\right. & \left.B(-\mu))\left(|g(\mu)|^{2}-|g(-\mu)|^{2}\right) \log (-z)\right] G_{0}(z, \mu+\delta \mu)= \\
= & {\left[1-\frac{\lambda^{2}}{4 \mu}\left(1-e^{-2 \pi \mu}\right)|g(-\mu)|^{2} \log (-z)\right] G_{0}(z, \mu+\delta \mu) }
\end{aligned}
$$

We see that besides the infrared renormalization of mass, which we ignore in the present paper, the bare Green's function is multiplied by the function of $\log (-z)$. Thus, even in Poincare patch, infrared corrections do appear when the two points are separated by a large geodesic distance. It would be interesting to understand the consequences of this result for the inflationary models in Poincare patch.

\section{Secular interactions and leading logarithms, complete $d S$ space}

In order to describe the global $d S$ space, we use the standard metric $d s^{2}=d t^{2}-\cosh ^{2} t\left(d \Omega_{d}\right)^{2}$. The eigenmodes for the Bunch-Davies vacuum are inherited from the sphere. To simplify notations we write them for $d=1$ :

$$
f_{q}(t) \propto P_{-\frac{1}{2}+i \mu}^{-q}(i \sinh t)
$$

where $q$ is an integer. These modes are selected by the condition that they are regular when continued to the southern hemisphere $(t=-i \vartheta ; \vartheta>0)$.

${ }^{4}$ To derive this formula we can make a Fourier transform

$$
\int^{\frac{\mu}{\tau}} d q \cdot \tau\left[A(\mu) A^{*}(-\mu) \Gamma(q \tau)^{2 i \mu}+A(-\mu) A^{*}(\mu) \Gamma^{*}(q \tau)^{-2 i \mu}+\left(|A(\mu)|^{2}+|A(-\mu)|^{2}\right) C\right] e^{i q x}
$$

and retain only terms of the order $\lambda^{2} \log (-z)$ while neglecting the terms of the order $\lambda^{2}$. 
The logarithmic divergences appear when $|q| \gg 1$ and $|t| \rightarrow \infty$. In this regions the Legendre functions can be replaced by the Bessel functions. We have:

$$
f_{q}(t) \underset{q \rightarrow \infty}{\longrightarrow}\left\{\begin{array}{l}
\tau^{d / 2} h(q \tau), \quad \tau=e^{-t}, \quad t \rightarrow \infty \\
\widetilde{\tau}^{d / 2} h^{*}(q \widetilde{\tau}), \quad \widetilde{\tau}=e^{+t}, \quad t \rightarrow-\infty .
\end{array}\right.
$$

As it should be, this is exactly the doubled Poincare patch.

Let us use these modes to calculate perturbative corrections to $\left\langle\varphi^{2}(n)\right\rangle$, assuming that the interaction begins adiabatically in the far past, with $\widetilde{\tau}=\varepsilon \rightarrow 0$, while the "observer" sits in the future at fixed $\tau$. The most important contribution comes from the +- term in the Fig.5. We

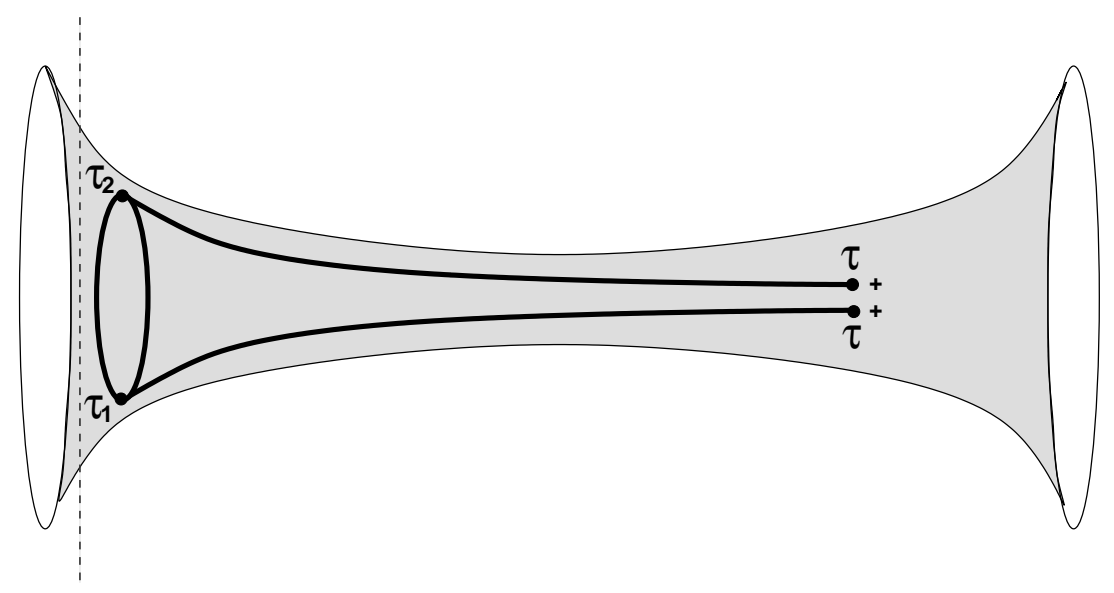

Figure 5: Relevant diagram, leading to IR divergence, in complete $d S$ space.

have

$$
\left\langle\varphi^{2}(n)\right\rangle^{(1)}=\lambda^{2} \tau^{d} \int \frac{d^{d} q}{(2 \pi)^{d}}|h(q \tau)|^{2} \int_{\varepsilon}^{\infty} \frac{d \widetilde{\tau}_{1} d \widetilde{\tau}_{2}}{\widetilde{\tau}_{1} \widetilde{\tau}_{2}}\left(h^{*}\left(q \widetilde{\tau}_{1}\right) h\left(q \widetilde{\tau}_{2}\right)\right) \cdot\left(\widetilde{\tau}_{1} \widetilde{\tau}_{2}\right)^{\frac{d}{2}} \cdot \sigma_{q}\left(\widetilde{\tau}_{1}, \widetilde{\tau}_{2}\right)
$$

where

$$
\sigma_{q}\left(\widetilde{\tau}_{1}, \widetilde{\tau}_{2}\right)=\int \frac{d^{d} k}{(2 \pi)^{d}} h^{*}\left(k \widetilde{\tau}_{1}\right) h\left(k \widetilde{\tau}_{2}\right) \cdot h^{*}\left(|k-q| \widetilde{\tau}_{1}\right) h\left(|k-q| \widetilde{\tau}_{2}\right) .
$$

We consider here only the dominant contribution, when $t_{1}, t_{2}$ are both in the far past. If $k \gg q$, we get the following scaling property:

$$
\sigma_{q}\left(\widetilde{\tau}_{1}, \widetilde{\tau}_{2}\right) \approx \sigma_{0}\left(\widetilde{\tau}_{1}, \widetilde{\tau}_{2}\right)=\left(\widetilde{\tau}_{1} \widetilde{\tau}_{2}\right)^{-d / 2} \Phi\left(\frac{\widetilde{\tau}_{1}}{\widetilde{\tau}_{2}}\right) .
$$

The integral (16) becomes:

$$
\begin{array}{r}
\left\langle\varphi^{2}(n)\right\rangle^{(1)}=\lambda^{2} \tau^{d} \int \frac{d^{d} q}{(2 \pi)^{d}}|h(q \tau)|^{2} \int_{\varepsilon}^{\infty} \frac{d \widetilde{\tau}_{1} d \widetilde{\tau}_{2}}{\widetilde{\tau}_{1} \widetilde{\tau}_{2}}\left(B(\mu)\left(\frac{\widetilde{\tau}_{1}}{\widetilde{\tau}_{2}}\right)^{i \mu}+B(-\mu)\left(\frac{\widetilde{\tau}_{1}}{\widetilde{\tau}_{2}}\right)^{-i \mu}\right) \Phi\left(\frac{\widetilde{\tau}_{1}}{\widetilde{\tau}_{2}}\right)= \\
=\text { const } \cdot \lambda^{2} \cdot \tau^{d} \int d^{d} q|h(q \tau)|^{2} \log \left(\frac{\mu}{q \varepsilon}\right) .
\end{array}
$$

The UV divergence at large $q$ must be cut-off by the condition $q \tau \lesssim M_{p l}$. Thus we get the result

$$
\left\langle\varphi^{2}(n)\right\rangle^{(1)}=\text { const } \cdot \lambda^{2} M_{P l}^{d-1} \log \left(\frac{\mu}{M_{P l}} \frac{\tau}{\varepsilon}\right)=\text { const } \cdot \lambda^{2}\left\langle\varphi^{2}(n)\right\rangle^{(0)} \cdot \log \left(\frac{\mu \tau}{M_{p l} \varepsilon}\right) .
$$


This formula is valid if:

$$
\varepsilon \ll \frac{\mu}{M_{P l}} \tau
$$

which means that the time $T$ during which the interaction was on, satisfies

$$
T=\frac{1}{H} \log \left(\frac{\tau}{\varepsilon}\right) \gg \frac{1}{H} \log \left(\frac{M_{P l}}{m}\right)
$$

(where we reinstated the Hubble constant).

In the Schwinger - Keldysh language we accounted for the $(+/-)$ self-energy part. There are, of course other insertions, $(+/+)$ and $(-/-)$, also generating secular logarithms. However, they are proportional to $\int d^{d} q h^{2}(q \tau)$ and its conjugate. This integral is UV convergent due to the oscillations of $h(q \tau)$. Hence there are no UV/IR mixing in these terms and their secular contribution, while non-zero, does not contain $M_{p l}$, unlike (17).

It is also instructive to write the above correction in the covariant form. Various SchwingerKeldysh propagators are expressed in terms of the different boundary values of a single analytic Wightman function, $g\left(n \cdot n^{\prime}\right)$, e.g. $G_{++}=g\left(n \cdot n^{\prime}-i 0\right), G_{+-}=g\left(n \cdot n^{\prime}+i \epsilon \operatorname{sgn}\left(n_{0}-n_{0}^{\prime}\right)\right)$ etc. The function $g(z)$ is real for $z \leq 1$ which corresponds to the space-like separations. By combining terms in the Schwinger-Keldysh diagrams it is easy to get $\left\langle\varphi^{2}\right\rangle=\left\langle\varphi^{2}\right\rangle_{I}+\left\langle\varphi^{2}\right\rangle_{I I}$ wherf 5

$$
\begin{array}{r}
\left\langle\varphi^{2}\right\rangle_{I} \sim \int^{n_{0}} d n_{1} d n_{2} \operatorname{Im}\left(g\left(n n_{1}\right)\right) \operatorname{Im}\left(g\left(n n_{2}\right)\right)\left\langle\left\{J\left(n_{1}\right), J\left(n_{2}\right)\right\}\right\rangle \\
\left\langle\varphi^{2}\right\rangle_{I I} \sim-2 i \int^{n_{0}} d n_{1} d n_{2} \theta\left(n_{10}-n_{20}\right) \operatorname{Im}\left(g\left(n n_{1}\right)\right) \operatorname{Re}\left(g\left(n n_{2}\right)\right)\left\langle\left[J\left(n_{1}\right), J\left(n_{2}\right)\right]\right\rangle
\end{array}
$$

where $J \sim \varphi^{2}(n)$. The first term represents the contribution of the real particles created from the vacuum, while the second term comes from the virtual particles. The logarithmic divergence arises from the domain where $n_{10}, n_{20} \rightarrow-\infty$, while $\left(n_{1} n_{2}\right) \sim 1$. The mathematical origin of the UV/IR mixing lies in the fact that the first integrand contains terms $g(z+i 0) g\left(z^{\prime}-i 0\right)$ which become singular on the light cone while infrared divergent in the infinite past. This phenomenon never happens in the Minkowski space.

In higher orders there are higher powers of the logarithms. Their summation requires the kinetic equation and will be discussed elsewhere.

\section{Conclusions}

The physical interpretation of the above estimates is the following. We are considering a complete $d S$ space. All points of this space are equivalent, so that the statements that at a given point we have expansion or contraction are meaningless. However, if we fix the position of the observer, one can define domains, such that the signal sent from them will be either red shifted or blue shifted. The essence of the formula (17) can be grasped from the Fig.5. We integrate the interaction over the faraway past region. The size of the loop determines the interaction scale $\sim 1 / m$, which is a large quantity. While the signal from the interaction region propagates along the geodesics to the observer, sitting at the point $\tau$, it is blue-shifted to the Planck scale $\sim 1 / M_{p l}$. As a result we get

\footnotetext{
${ }^{5}$ The easiest way to derive these formulas is to use the definition of (anti)chronological products

$T\left(J_{1} J_{2}\right)=\frac{1}{2}\left\{J_{1}, J_{2}\right\}+\sigma \frac{1}{2}\left[J_{1}, J_{2}\right], \quad \widetilde{T}\left(J_{1} J_{2}\right)=\frac{1}{2}\left\{J_{1}, J_{2}\right\}-\sigma \frac{1}{2}\left[J_{1}, J_{2}\right], \quad J_{1} J_{2}=\frac{1}{2}\left\{J_{1}, J_{2}\right\}+\frac{1}{2}\left[J_{1}, J_{2}\right]$,

where $\sigma=\operatorname{sign}\left(n_{10}-n_{20}\right)$ and then use the symmetry of the measure w.r.t. intrchange of $1 \leftrightarrow 2$ to reduce the integration domain to the region $n_{10}>n_{20}$. All the terms containing anticommutator of currents are collected into $\left\langle\varphi^{2}\right\rangle_{I}$, all the terms containing commutator are in $\left\langle\varphi^{2}\right\rangle_{I I}$.
} 
a very curious UV/IR mixing. In the flat space we expect that UV and IR divergencies contribute to the physical quantities independently - we do not expect the terms, like (17), which are both UV and IR divergent at the same time. This is a specific feature of the curved space.

The $\varepsilon$ dependence of the physical matrix element discussed above indicates a breakdown of the $d S$ symmetry; as always, spontaneous symmetry breaking manifests itself through the sensitive dependence on the boundary conditions. The logarithms will be present even for a patch of the $d S$ space, provided that it is "large", that is the past cone of the observer intersects a decent portion of the past infinity. As we saw from Fig.4, this is not the case for the Poincare patch; for it "the world is not enough".

Let us explain our motives for using the global $d S$ space, while in the inflationary theories only a small part of it is usually present. Our goal is to resolve the puzzle of the cosmological constant by infrared means. We start with the Einstein action with the cosmological constant present. The standard procedure in field theory is to assume first that we can neglect quantum corrections at large distances, find a classical solution and then evaluate the corrections. It is this procedure which allows us to use classical Einstein or Navier - Stokes and forbids the similar use of the Yang - Mills equations (due to asymptotic freedom) and sometimes the diffusion equation (due to Anderson's localization).

In such a setting we must consider the global $d S$ space as a first step. If a starting point were incomplete space, we would end up with the unitarity problem, since the particles can disappear from the space. Of course it is possible to have a Poincare patch glued to the Minkowski one in such a way that the result is geodesically complete. However this space will not be a solution of the Einstein equations with the cosmological constant only. It is also possible to modify the Einstein action so that we have a different background without IR divergences. This looks ambiguous and is far from our goal, which is to tame infrared divergencies. We should remember that to solve the $\Lambda$-problem one must be searching for the infrared effects and not running from them. IR divergence is not a problem but an opportunity.

Another question is related to the choice of the Bunch-Davies vacuum in the above calculation. What is the reason for this (apart from the tradition)? It seems that the right starting point should be the state with the longest life time. We haven't proved that this is the case, but various estimates make us believe that the Bunch-Davies vacuum is the most stable one. In the appendix we present the propagators for the different possible ground states. It should not be difficult to extend our analysis to other vacua.

Finally, there is a number of valuable papers [15] intersecting with our work, but it seems that our approach brought some new and unusual results.

\section{Acknowledgements}

We would like to thank E.Akhmedov, J.Maldacena and V.Mukhanov for useful discussions. This work was supported in part by the NSF grant number PHY-0756966.

\section{Appendix A. Oscillator, relative probabilities}

Here we briefly discuss some ideas mentioned in the main text using the simplest model - quantum mechanical particle. Take an oscillator with variable frequency

$$
\left(\partial_{t}^{2}+m^{2}+U(t)\right) \varphi=0
$$


Let us introduce Jost functions

$$
\begin{array}{rlrl}
f_{\text {in }}(t) & \rightarrow \frac{1}{\sqrt{2 m}} e^{i m t} & t & \rightarrow-\infty \\
f_{\text {out }}(t) & \rightarrow \frac{1}{\sqrt{2 m}} e^{i m t} & t & \rightarrow+\infty
\end{array}
$$

As well known in scattering theory

$$
f_{\text {in }}(t)=\alpha f_{\text {out }}(t)+\beta f_{\text {out }}^{*}(t)
$$

$|\alpha|^{2}-|\beta|^{2}=1$. Let us find the vacuum decaying amplitude. We define the (in) and (out) vacua in a usual way:

$$
\varphi=a f_{\text {in }}^{*}+a^{\dagger} f_{\text {in }}=b f_{\text {out }}^{*}+b^{\dagger} f_{\text {out }}
$$

and $a|0\rangle_{\text {in }}=b|0\rangle_{\text {out }}=0,\left[a, a^{\dagger}\right]=\left[b, b^{\dagger}\right]=1$. The in/out Green's function

$$
G={ }_{\text {out }}\left\langle 0\left|T \varphi\left(t_{1}\right) \varphi\left(t_{2}\right)\right| 0\right\rangle_{\text {in }} \frac{1}{{ }_{\text {out }}\langle 0 \mid 0\rangle_{\text {in }}}=\frac{1}{\alpha} f_{\text {in }}\left(t_{<}\right) f_{\text {out }}^{*}\left(t_{>}\right)
$$

This Green's function is the one satisfying the composition principle [1]

$$
\frac{\partial G\left(t_{1}, t_{2}\right)}{\partial m^{2}}=-i \int_{-\infty}^{\infty} G\left(t_{1}, t\right) G\left(t, t_{2}\right) d t
$$

This equation allows to represent $G$ in terms of the Feynman's sum over paths. The amplitude to produce $2 n$-particles is given by:

$$
A_{0 \rightarrow 2 n}={ }_{\text {out }}\left\langle 0\left|\frac{b^{2 n}}{\sqrt{2 n !}}\right| 0\right\rangle_{\text {in }}={ }_{\text {out }}\langle 2 n \mid 0\rangle_{\text {in }}
$$

Let us express it in terms of the Green functions:

$$
G\left(t_{1}, \ldots, t_{2 n}\right)=\frac{{ }_{\text {out }}\left\langle 0\left|T \varphi\left(t_{1}\right) \ldots \varphi\left(t_{2 n}\right)\right| 0\right\rangle_{\text {in }}}{\text { out }\langle 0 \mid 0\rangle_{\text {in }}} \rightarrow_{t_{j} \rightarrow \infty} \frac{{ }_{\text {out }}\left\langle 0\left|b^{2 n}\right| 0\right\rangle_{\text {in }}}{\text { out }\langle 0 \mid 0\rangle_{\text {in }}} \frac{\left(e^{-i m \sum t_{j}}\right)}{(2 m)^{n}}+\ldots
$$

The Wick theorem on another hand gives

$$
G\left(t_{1}, \ldots t_{2 n}\right)=G\left(t_{1}, t_{2}\right) \ldots G\left(t_{2 n-1}, t_{2 n}\right)+\text { perm. }
$$

Total number of permutations is $(2 n-1)(2 n-3) \ldots=(2 n-1) ! !=\frac{(2 n) !}{n ! 2^{n}}$. From here we derive

$$
\left|\frac{A_{0 \rightarrow 2 n}}{A_{0 \rightarrow 0}}\right|=\frac{(2 n) !}{2^{n} n !}\left(\frac{\beta}{\alpha}\right)^{n} \frac{1}{\sqrt{(2 n) !}}
$$

(we pick up the terms containing $e^{-i m \sum t_{j}}$ from the products of the Green's functions). Hence the probability to produce $2 n$ particles is

$$
\frac{W_{2 n}}{W_{0}}=\frac{\left.\left.\right|_{\text {out }}\langle 2 n \mid 0\rangle_{\text {in }}\right|^{2}}{\left.\operatorname{lout}_{\text {out }}\langle 0 \mid 0\rangle_{\text {in }}\right|^{2}}=\frac{(2 n) !}{(n !)^{2} 4^{n}}\left|\frac{\beta}{\alpha}\right|^{2 n}
$$

The normalization condition gives

$$
1=W_{0}+\sum_{n=1}^{\infty} W_{2 n}=W_{0}\left(1+\sum_{n=1}^{\infty} \frac{W_{2 n}}{W_{0}}\right)=W_{0} \sum_{n=0}^{\infty} \frac{(2 n) !}{(n !)^{2} 4^{n}}\left|\frac{\beta}{\alpha}\right|^{2 n}=\frac{W_{0}}{\sqrt{1-\left|\frac{\beta}{\alpha}\right|^{2}}}
$$


Thus $W_{0}=\sqrt{1-\left|\frac{\beta}{\alpha}\right|^{2}}=\frac{1}{|\alpha|}$. It is interesting to note that the Green functions give relative probabilities, $P_{0 n}=\frac{W_{n}}{W 0}$. When the vacuum is unstable $W_{0}<1$ and hence $\sum_{n} P_{0 n}>1$. This may open the way to interpretation of the non-unitary field theories - they describe unstable vacua. For example in non-unitary CFT there are well classified operators with negative norms. The 'probabilities' extracted from the Green functions satisfy $\sum_{n} \pm P_{n}=1, \sum_{n} P_{n}>1$ and $P_{n}$ can be interpreted as relative probabilities.

Another interesting relation is $W_{0}=e^{-\Gamma}, \frac{\partial \Gamma}{\partial m^{2}}=\int \operatorname{Im} G(t, t) d t=\operatorname{Im}\left(\frac{\beta}{\alpha} \int f_{\text {out }}^{*}(t)^{2} d t\right)$. We see that $\operatorname{Im} G(t, t) \neq 0$ signals vacuum instability. The typical back-reaction of produced particles on the field $U(t)$ is characterized by the 'current' $J={ }_{i n}\left\langle 0\left|\varphi(t)^{2}\right| 0\right\rangle_{i n}$. We see that

$$
J(t)=\left|f_{\text {in }}(t)\right|^{2}
$$

and (omitting rapidly oscillating terms)

$$
J(\infty)-J(-\infty)=\frac{1}{2 m}\left(|\alpha|^{2}+|\beta|^{2}-1\right)=\frac{1}{m}|\beta|^{2}
$$

Thus, the back-reaction does not become large with time. We can also evaluate the average number of the produced excitations $\bar{n}=\sum W_{2 n}(2 n)=|\beta|^{2}$. However it is to be remembered that this number fluctuates: $\overline{(n-\bar{n})^{2}} \sim \bar{n}$.

\section{Appendix B. The Green's functions of the de Sitter space.}

The propagator is a solution to the inhomogeneous Klein-Gordon equation with a delta-function source. Such solution is not unique and defined modulo a solution to homogeneous equation. In the flat Minkowski space there is a well established prescription of doing integration in the complex momentum plane which fixes this ambiguity and uniquely defines the propagators. This prescription however is not straightforwardly generalized to curved space which we are working with, hence we need a different setup. A convenient method is to write the propagator in the form

$$
G\left(t_{1}, t_{2}\right)=\frac{1}{W\left[\varphi_{1}, \varphi_{2}\right]} \varphi_{1}\left(t_{<}\right) \varphi_{2}\left(t_{>}\right)
$$

Where $\varphi_{1,2}(t)$ are two linearly independent solutions to the homogeneous equation, $W\left[\varphi_{1}, \varphi_{2}\right]$ is their Wronskian and $t_{<>}$is the smallest and largest of the times $t_{1,2}$. The above mentioned ambiguity is reflected here in the different possible choices for the solutions to the homogeneous equation $\varphi_{1,2}(t)$. This choice depends on the problem we want to solve. In case of in-out propagator
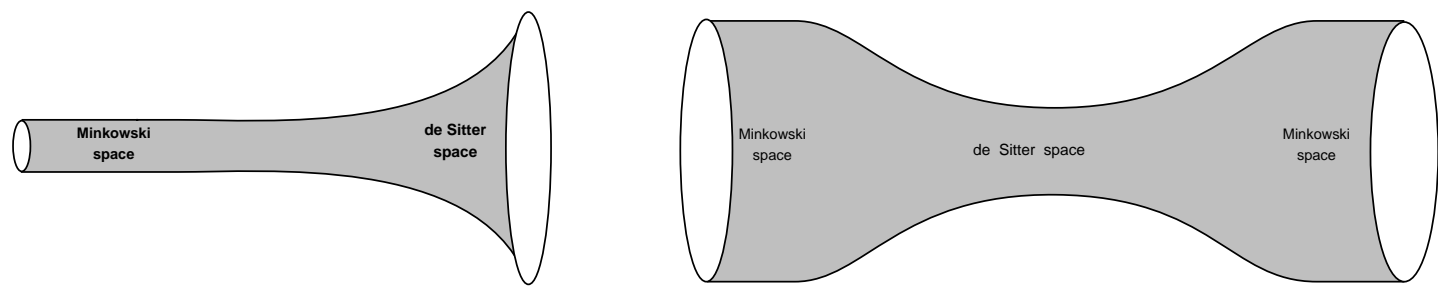

Figure 6: On the left - geometry of expanding Universe, on the right - geometry of complete de Sitter space.

we should choose $\varphi_{1,2}(t)$ to be Jost functions of the scattering problem, i.e. $\varphi_{1}$ to be a plane wave 
at $t \rightarrow-\infty$ and $\varphi_{2}$ to be a plane wave at $t \rightarrow+\infty$. In case of in-in propagator both $\varphi_{1}$ and $\varphi_{2}$ should be plane waves at $t \rightarrow-\infty$.

Another complication comes from the fact that a de Sitter patch can be embedded into flat space in numerous ways. Below we illustrate this by considering two possibilities: initially flat space adiabatically starts to expand; and complete de Sitter space glued with two cylinders on both plus and minus time infinities (see Fig.6). The Green's functions for these geometries turn out to be very different.

\subsection{First geometry, Poincare patch}

We start with the first geometry, which can be conveniently described by the Poincare patch $d s^{2}=\left(\frac{\alpha}{\tau}\right)^{2}\left(d \tau^{2}-d x^{2}\right)$ and for simplicity we work in $d=1$ dimensions. The wave equation for a mode with comoving momentum $p$ can be written as

$$
\phi_{\tau \tau}+p^{2} \phi+\frac{(m \alpha)^{2}}{\tau^{2}} \phi=0
$$

with $\nu=i \mu=i \sqrt{(m \alpha)^{2}-\frac{1}{4}}$. It's solution can be written in terms of Bessel functions. In the in-region the Jost functions are Hankel functions, while in the out region they are Bessel functions. Thus, according to (19) we obtain

$$
G_{P}^{\text {in } / \text { out }}(1,2)=\int_{0}^{\infty} d p \cos \left[p\left(x_{2}-x_{1}\right)\right] \sqrt{\tau_{1} \tau_{2}} H_{\nu}^{(1)}\left(p \tau_{>}\right) J_{\nu}\left(p \tau_{<}\right)
$$

for in-out (Feynman) propagator and

$$
G_{P}^{i n / i n}(1,2)=\int_{0}^{\infty} d p \cos \left[p\left(x_{2}-x_{1}\right)\right] \sqrt{\tau_{1} \tau_{2}} H_{\nu}^{(1)}\left(p \tau_{>}\right) H_{\nu}^{(2)}\left(p \tau_{<}\right)
$$

for in-in propagator. Integrals w.r.t. momentum can be carried out explicitly by using 6.672.4, 6.672 .3 of [14]. The result is

$$
\begin{gathered}
G_{P}^{\text {in } / \text { out }}(1,2)=\mathcal{Q}_{\nu-\frac{1}{2}}(z+i 0) \\
G_{P}^{\text {in } / \text { in }}(1,2)=\frac{1}{\cos (\pi \nu)} \mathcal{P}_{\nu-\frac{1}{2}}(-z-i 0)
\end{gathered}
$$

The first expression was suggested in [1], the second one is the Bunch-Davies propagator. Both are expressed in terms of geodesic distance

$$
z=\frac{\tau_{1}^{2}+\tau_{2}^{2}-\left(x_{2}-x_{1}\right)^{2}}{2 \tau_{1} \tau_{2}}
$$

To make the integrals (20), (21) convergent we need to shift $\tau_{>}$into complex plane. This shift together with $\frac{\partial z}{\partial \tau_{>}}>0$ determines $i 0$ prescription in the arguments of Legendre functions.

Another nice representation of in-out propagator can be obtained from 6.669.3-4 of [14]

$$
G_{P}^{\text {in/out }}(1,2)=\int_{0}^{\infty} d p \cos \left[p x_{21}\right] \sqrt{\tau_{1} \tau_{2}} e^{-i \pi \nu} \int_{0}^{\infty} \frac{d s}{\sinh s} e^{i p\left(\tau_{1}+\tau_{2}\right) \operatorname{coth} s} J_{2 \nu}\left(\frac{2 p \sqrt{\tau_{1} \tau_{2}}}{\sinh s}\right)
$$


This expression are analogous to Schwinger proper time representation of the Feynman propagator in electric field. It's path integral derivation can be found in [13. It is also convenient for the calculation of the imaginary part of in-out propagator at coincident points, which is related to the imaginary part of effective action. Taking the limit of coincident points in (24) and integrating over $k$ by using 6.611 .1 we get up to inessential constant

$$
\operatorname{Im}\left[G_{P}^{\text {in/out }}(1,1)\right]=\operatorname{Im} \frac{-1}{2 \pi} \int_{-\infty}^{+\infty} \frac{d s}{\sinh s} e^{2 i \mu s}=-\sum_{n=1}^{\infty}(-1)^{n} e^{-2 \pi n \mu}=\frac{e^{-2 \pi \mu}}{1+e^{-2 \pi \mu}}
$$

Non-vanishing of this quantity signals instability of the vacuum w.r.t. creation of particles, similarly to Schwinger mechanism in constant electric field.

\subsection{Geometry of the Complete de Sitter space}

The second geometry can be described by a metric

$$
d s^{2}=d t^{2}-\cosh t^{2} d \varphi^{2}
$$

with compact coordinate $\varphi \in[0,2 \pi]$. The wave equation for a mode with integer momentum $p$ can be written as

$$
\ddot{\phi}+\tanh t \dot{\phi}+m^{2} \phi+\frac{p^{2}}{\cosh t^{2}} \phi=0
$$

The general solution of this equation is 6

$$
\phi=\frac{1}{\sqrt{\cosh t}}\left[C_{1} \mathrm{P}_{p-\frac{1}{2}}^{ \pm \nu}( \pm \tanh t)+C_{2} \mathrm{Q}_{p-\frac{1}{2}}^{ \pm \nu}( \pm \tanh t)\right]
$$

Choosing the Jost functions and plugging them into (19) we obtain

$$
\begin{gathered}
G_{d S}^{i n / \text { in }}=\frac{\pi}{2 \sqrt{\cosh t_{1} \cosh t_{2}}} \sum_{p=0}^{\infty} \varepsilon_{p} \cos (p \varphi) \mathrm{P}_{p-\frac{1}{2}}^{-\nu}\left(-\tanh t_{>}\right) \mathrm{P}_{p-\frac{1}{2}}^{\nu}\left(-\tanh t_{<}\right) \\
G_{d S}^{\text {in } / \text { out }}=\frac{1}{\sqrt{\cosh t_{1} \cosh t_{2}}} \sum_{p=0}^{\infty} \varepsilon_{p} \Gamma\left(\nu-p+\frac{1}{2}\right) \Gamma\left(\nu+p+\frac{1}{2}\right) \cos (p \varphi) \mathrm{P}_{p-\frac{1}{2}}^{-\nu}\left(\tanh t_{>}\right) \mathrm{P}_{p-\frac{1}{2}}^{-\nu}\left(-\tanh t_{<}\right)= \\
=\frac{2}{\cos (\pi \nu) \sqrt{\cosh t_{1} \cosh t_{2}}} \sum_{p=0}^{\infty} \varepsilon_{p} \cos (p \varphi) \mathrm{P}_{p-\frac{1}{2}}^{-\nu}\left(\tanh t_{>}\right) \mathrm{Q}_{p-\frac{1}{2}}^{\nu}\left(\tanh t_{<}\right)
\end{gathered}
$$

where $\varepsilon_{p}=1$ for $p=0$ and $\varepsilon_{p}=2$ otherwise. It is possible to sum up the $p$-series and express the result in terms of Lorentz invariant quantities (geodesic distance $z$ and $\sigma=\operatorname{Sign}\left[n_{0}(1)+n_{0}(2)\right]$ ).

$$
G_{d S}^{i n / i n}=\frac{1}{2 i}\left[\mathcal{Q}_{\nu-\frac{1}{2}}(-z-i \varepsilon)-\mathcal{Q}_{\nu-\frac{1}{2}}(-z+i \varepsilon)\right]-\frac{\pi}{4 i \cos (\pi \nu)}(\sigma+1)\left[\mathcal{P}_{\nu-\frac{1}{2}}(z+i \varepsilon)-\mathcal{P}_{\nu-\frac{1}{2}}(z-i \varepsilon)\right]
$$

We would like to emphasize that this expression is Lorentz invariant, since functions $\mathcal{P}_{\nu-\frac{1}{2}}(z \pm i \varepsilon)$ have a cut only for $z<-1$, but $\sigma$ is Lorentz invariant quantity for $z<-1$. Also, this function

\footnotetext{
${ }^{6}$ In the following we adopt the notation $\mathrm{P}_{\nu}^{\mu}(x)$ and $\mathrm{Q}_{\nu}^{\mu}(x)$ for associated Legendre functions on the cut when the argument is $-1<x<1$. These functions are defined by [14] 8.702-8.705. For Legendre functions in the complex plane we use symbols $\mathcal{P}_{\nu}^{\mu}(z)$ and $\mathcal{Q}_{\nu}^{\mu}(z)$.
} 
vanishes within the light-cone of the past of the antipod7. This area corresponds to $z<-1$, $\sigma=-1$, see Fig.7.

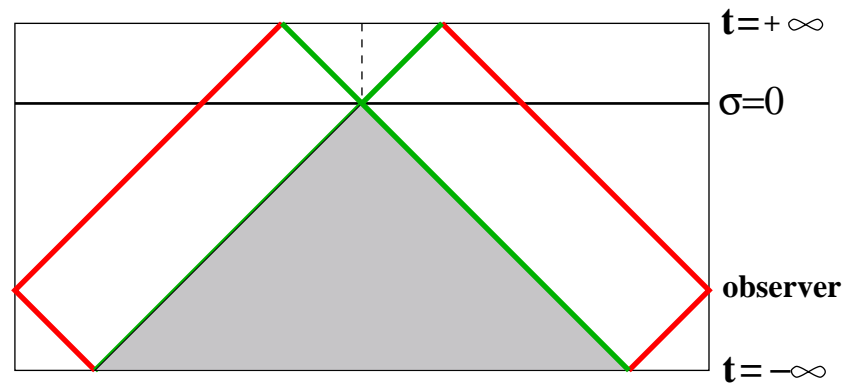

Figure 7: Conformal diagram. Red - the light cone of the observer, green - the light cone of the antipodal point. Gray - the area where $G_{d S}^{i n / i n}=0$.

For the Feynman propagator we obtain

$$
G_{d S}^{\text {in/out }}=\frac{1}{\cos (\pi \nu)}\left[\mathcal{Q}_{\nu-\frac{1}{2}}(-z-i \varepsilon)+\mathcal{Q}_{\nu-\frac{1}{2}}(-z+i \varepsilon)\right]
$$

Note, that this answer is different from in-out propagator in Poincare patch (22), c.f. also [11]. It also has a non-vanishing imaginary part at coincident points.

\section{References}

[1] A. M. Polyakov, "De Sitter Space and Eternity," Nucl. Phys. B 797 (2008) 199 arXiv:0709.2899 [hep-th]].

[2] A. M. Polyakov, "Decay of Vacuum Energy," arXiv:0912.5503 [hep-th].

[3] I. Antoniadis, P. O. Mazur and E. Mottola, "Cosmological dark energy: Prospects for a dynamical theory," New J. Phys. 9, 11 (2007) arXiv:gr-qc/0612068.

[4] A. I. Nikishov, "Pair production by a constant external field," Zh. Eksp. Teor. Fiz. 57, 1210 (1969).

N. B. Narozhnyi and A. I. Nikishov, "The Simplist processes in the pair creating electric field," Yad. Fiz. 11, 1072 (1970) [Sov. J. Nucl. Phys. 11, 596 (1970)].

[5] Y. Kluger, J. M. Eisenberg, B. Svetitsky, F. Cooper and E. Mottola, "Pair production in a strong electric field," Phys. Rev. Lett. 67, 2427 (1991).

Y. Kluger, J. M. Eisenberg, B. Svetitsky, F. Cooper and E. Mottola, "Fermion Pair Production In A Strong Electric Field," Phys. Rev. D 45, 4659 (1992).

[6] V. N. Gribov, "Local confinement of charge in massless QED," Nucl. Phys. B 206, 103 (1982).

\footnotetext{
${ }^{7}$ It could be convenient to cover the manifold $n_{0}^{2}-n_{1}^{2}-n_{2}^{2}=1$ by coordinates

$$
\left\{\begin{array}{l}
n_{0}=\cot \theta \\
n_{1}=\frac{\cos \varphi}{\sin \theta} \\
n_{2}=\frac{\sin \varphi}{\sin \theta}
\end{array} \quad z=\frac{\cos \left(\varphi_{1}-\varphi_{2}\right)-\cos \theta_{1} \cos \theta_{2}}{\sin \theta_{1} \sin \theta_{2}} \quad \sigma=\operatorname{Sign}\left[\sin \left(\theta_{1}+\theta_{2}\right)\right]\right.
$$
}


[7] E. T. Akhmedov and P. Burda, "A simple way to take into account back reaction on pair creation," Phys. Lett. B 687, 267 (2010) [arXiv:0912.3435 [hep-th]].

[8] S. P. Gavrilov and D. M. Gitman, "One-loop energy-momentum tensor in QED with electric-like background," Phys. Rev. D 78, 045017 (2008) arXiv:0709.1828 [hep-th]].

[9] I. D. Novikov and A. A. Starobinsky, "Quantum electrodynamic effects inside a charged bleack hole and the problem of cauchy horizons," Sov. Phys. JETP 51 (1980) 1 [Zh. Eksp. Teor. Fiz. 78 (1980) 3].

[10] G. E. Volovik, "Particle decay in de Sitter spacetime via quantum tunneling," JETP Lett. 90, 1-4 (2009). arXiv:0905.4639 [gr-qc]].

G. E. Volovik, "On de Sitter radiation via quantum tunneling," arXiv:0803.3367 [gr-qc]].

R. Di Criscienzo, L. Vanzo, S. Zerbini, "Applications of the Tunneling Method to Particle Decay and Radiation from Naked Singularities," JHEP 1005, 092 (2010). arXiv:1001.4617 [gr-qc]].

[11] E. T. Akhmedov, "Real or Imaginary? (On pair creation in de Sitter space)," Mod. Phys. Lett. A25, 2815-2823 (2010). [arXiv:0909.3722 [hep-th]].

[12] D. Marolf, I. A. Morrison, "The IR stability of de Sitter: Loop corrections to scalar propagators," [arXiv:1006.0035 [gr-qc]].

S. Hollands, "Correlators, Feynman diagrams, and quantum no-hair in deSitter spacetime," arXiv:1010.5367 [gr-qc]].

[13] F. Steiner, "Space-Time Transformations In Radial Path Integrals," Phys. Lett. A 106 (1984) 356.

[14] Gradshtein, Ryzhik. Table of integrals, series and products.

[15] M. Sasaki, H. Suzuki, K. Yamamoto and J. Yokoyama, "Superexpansionary divergence: Breakdown of perturbative quantum field theory in space-time with accelerated expansion," Published in Class.Quant.Grav.10:L55-L60,1993.

M. Bander, "Instability of $(1+1)$ de sitter space in the presence of interacting fields," Phys. Rev. D 82, 024003 (2010) arXiv:1003.3963 [hep-th]].

M. Bander, "Anomaly breaking of de Sitter symmetry," arXiv:1011.0699 [hep-th].

M. Bander, "Breaking of de Sitter Symmetry," arXiv:1011.2241 [hep-th]].

S. P. Kim, "Vacuum Structure of de Sitter Space," arXiv:1008.0577 [hep-th]].

E. Alvarez, R. Vidal, "Comments on the vacuum energy decay," arXiv:1004.4867 [hep-th]l.

H. Kitamoto, Y. Kitazawa, "Boltzmann equation in de Sitter space," Nucl. Phys. B839, 552-579 (2010). arXiv:1004.2451 [hep-th]].

D. I. Podolsky, "On triviality of $\lambda \phi^{4}$ quantum field theory in four dimensions," arXiv:1003.3670 [hep-th]].

J. Bros, H. Epstein, U. Moschella, "Scalar tachyons in the de Sitter universe," Lett. Math. Phys. 93, 203-211 (2010). arXiv:1003.1396 [hep-th]].

J. Bros, H. Epstein, U. Moschella, "Particle decays and stability on the de Sitter universe," Annales Henri Poincare 11, 611-658 (2010). arXiv:0812.3513 [hep-th]].

E. T. Akhmedov, P. V. Buividovich, "Interacting Field Theories in de Sitter Space are NonUnitary," Phys. Rev. D78, 104005 (2008). arXiv:0808.4106 [hep-th]]. 
A. Higuchi, "Tree-level vacuum instability in an interacting field theory in de Sitter spacetime," Class. Quant. Grav. 26, 072001 (2009) arXiv:0809.1255 [gr-qc]].

G.E. Volovik, "Peculiarities in the dynamics of superfluid ${ }^{3} \mathrm{He}-\mathrm{A}$ : analog of chiral anomaly and of zero-charge," JETP 65, 1193 -1201 (1987). 